Audiology
Neurotology
Audiol Neurotol 2006;11(suppl 1):16-20

DOI: $\underline{10.1159 / 000095609}$
Received: January 23, 2006

Accepted after revision: July 12, 2006

Published online: October 6, 2006

\title{
A Frequency-Position Function for the Human Cochlear Spiral Ganglion
}

\author{
Divya Sridhar Olga Stakhovskaya Patricia A. Leake
}

Epstein Laboratory, Department of Otolaryngology-Head and Neck Surgery, University of California San Francisco,

San Francisco, Calif., USA

\section{Key Words}

Cochlear implants · Frequency map · Human cochlear

spiral ganglion $\cdot$ Critical bands

\begin{abstract}
Greenwood's frequency-position function for the organ of Corti $(\mathrm{OC})$ is commonly used to estimate represented frequencies for cochlear implant $(\mathrm{Cl})$ electrodes, both in temporal bone studies and in imaging studies of living $\mathrm{Cl}$ recipients. However, many contemporary Cls position stimulating electrodes near the modiolus, directly targeting the spiral ganglion (SG) cells within Rosenthal's canal. At the extreme base and apex, the SG does not extend as far as the $O C$, and the radial nerve fibers take a tangential course into the modiolus resulting in a potential offset between the frequency maps of the OC and SG. In this investigation, human cadaveric cochleae $(n=7)$ were studied in surface preparations after osmium staining. The OC and SG lengths were measured and radial fiber trajectories traced to identify frequency-matched points on each structure. These data allowed derivation of a mathematical function correlating represented frequency along the $\mathrm{OC}$ to position along the SG. A cubic function fit the data with a very high intersubject correlation. Better knowledge of the human SG 'neural frequency map' may help to refine electrode design, and to more accurately map $\mathrm{Cl}$ channel filter bands to the appropriate cochlear place along the SG, which may be advantageous for more sophisticated $\mathrm{Cl}$ outcomes, such as music apprecia-
\end{abstract}

tion. These data also could be valuable for electroacoustic stimulation, by defining the insertion distance of a $\mathrm{Cl}$ electrode required to reach specific frequencies (based upon preoperative imaging) in an individual subject, thus helping to avoid trauma to cochlear regions with residual hearing.

Copyright $\odot 2006$ S. Karger AG, Basel

\section{Introduction}

Characteristic frequencies for cochlear implant (CI) electrode stimulation sites usually are estimated with the Greenwood frequency-position equation [Greenwood, 1990], which has been used both in temporal bone studies [Kawano et al., 1996; Wardrop et al., 2005a, b] and in imaging studies of living CI recipients [Ketten et al., 1998; Skinner et al., 2002]. The original Greenwood frequencyposition function for the organ of Corti (OC) was derived from human frequency resolution-integration estimates (critical bandwidths), assuming that critical bandwidths followed an exponential function of distance along the cochlear partition and corresponded to a constant physical distance on the basilar membrane. Greenwood proposed the following equation for frequency along the $\mathrm{OC}$ : $\mathrm{F}=\mathrm{A} \cdot\left(10^{\mathrm{ax}}-\mathrm{k}\right)$, where $\mathrm{F}$ is frequency, $\mathrm{x}$ is the position on the basilar membrane; coefficients for human cochleae: $\mathrm{A}=165.4, \mathrm{a}=2.1$ (if $\mathrm{x}$ is expressed in proportion of the total basilar membrane length), $\mathrm{k}=0.88$ [Greenwood, 1990]. The equation allows the estimation of represented

\section{KARGER}

Fax +4161306 1234

E-Mail karger@karger.ch

www.karger.com (c) 2006 S. Karger AG, Basel

$1420-3030 / 06 / 0117-0016 \$ 23.50 / 0$

Accessible online at:

www.karger.com/aud
Patricia A. Leake, $\mathrm{PhD}$

Epstein Laboratory, Department of Otolaryngology-H.N.S

University of California San Francisco

533 Parnassus Ave., Room U490, San Francisco, CA 94143-0526 (USA)

Tel. +1 415476 5958, Fax +1 415476 2169, E-Mail leake@itsa.ucsf.edu 
frequency based on cochlear place and expressed as proportion of the total length of OC. However, OC length cannot be determined in most temporal bone and imaging studies, and estimates of frequency based upon the average OC length may be inaccurate due to individual variability. Moreover, some contemporary 'perimodiolar' CI electrodes (e.g. the Contour ${ }^{\mathrm{TM}}$ electrode from Cochlear Limited, Sydney, Australia; the HiFocus ${ }^{\mathrm{TM}}$ with positioner and the new Helix ${ }^{\mathrm{TM}}$ electrodes from Advanced Bionics Corporation, Sylmar, Calif., USA) are designed with stimulating sites facing the modiolus, and the spiral ganglion (SG) cell somata within Rosenthal's canal are the presumed target of excitation for such electrodes. The SG turns on a smaller radius and terminates sooner than the OC at both the basal and apical ends of the spiral. Therefore, represented frequency in the SG must differ from that of the OC, at least over part of its course. The goal of this study was to derive an accurate frequencyposition function for the human SG, based upon extrapolation from OC cochlear-place frequency and to explore the implications of the differences between SG and OC frequency maps with respect to the design and surgical insertion of CI.

\section{Materials and Methods}

Human temporal bones $(n=7)$ were harvested from fresh cadaver specimens within $24 \mathrm{~h}$ postmortem and fixed by immersion in $10 \%$ phosphate-buffered formalin. Only one cochlea (left or right) was studied from each cadaver, to better assess individual variability. The round window was opened as soon as possible to facilitate diffusion of the fixative into the cochlea. The labyrinth was isolated and the otic capsule bone was drilled away to 'blue line' the cochlea. Specimens were then stained with osmium tetroxide ( $1 \%$ in $0.1 \mathrm{M}$ phosphate buffer, $\mathrm{pH} 7.4$ ) to visualize the radial nerve fibers. The cochlea was microdissected to expose the cochlear duct, and a marker (small notch in the stria vascularis) was created in the upper basal coil at the point nearest to the vestibule (V). The specimens were decalcified for $24-36 \mathrm{~h}$ to further improve visibility of the radial nerve fibers within the osseous spiral lamina and then embedded in epoxy. Each cochlea was bisected through the modiolus, and the individual half-coils were removed from the block and remounted on a glass slide in a surface preparation that contained both the OC and adjacent SG. All cuts through the specimens were made using razor blades so that tissue loss was negligible. Digital images of each piece of the surface preparation were captured and calibrated by capturing an image of a stage micrometer at the same magnification and superimposing this image on the specimen images. The OC was measured directly in the digital images by tracing along its approximate center (along the tops of the pillar cells, if visible) beginning at the basal extreme of the basilar membrane and measuring each segment of the surface preparation (fig. 1). Semi-thin radial sections were cut at regular intervals along the cochlear spiral and

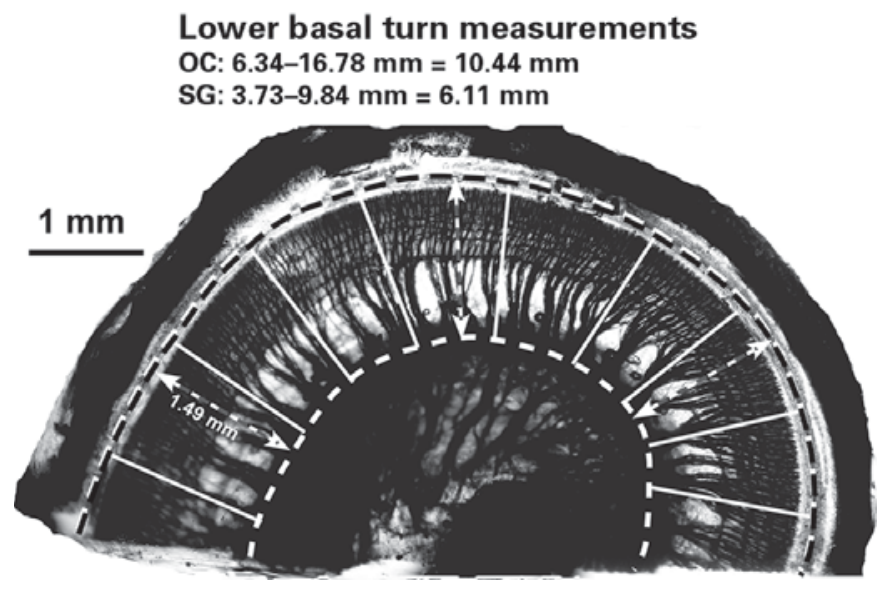

Fig. 1. Digital image of the lower basal turn of a human cochlea after epoxy embedding and surface preparation. The dashed white and black line indicates the $\mathrm{OC}$, which was $10.44 \mathrm{~mm}$ long in this sector of the cochlea. The white dashed arrows indicate how measurements from radial sections were used in digital images to define the center of the SG. The inner white dashed line marks the approximate center of the SG (length, $6.11 \mathrm{~mm}$ in this segment). The solid white lines denote the trajectories of radial nerve fibers, as drawn to define a series of frequency-matched points on the OC and SG.

used to define the distance from the OC to the center of the SG, to examine the morphology and locate the points at base and apex where SG terminates. Next, the SG was measured along the series of points defining its center in the digital images, and the trajectories of the radial nerve fibers were traced to define a series of frequency-matched coordinates along the OC and SG.

\section{Results}

As shown in table 1, the OC lengths measured in the calibrated digital images demonstrated considerable intersubject variability, and ranged from 30.5 to $36.87 \mathrm{~mm}$. The mean length of the $\mathrm{OC}$ was $33.31 \pm 2.38$ (SD) $\mathrm{mm}$ for the group of 7 cochlear specimens studied to date. In contrast, the average length of the SG was only $13.9 \pm$ $0.79 \mathrm{~mm}(\mathrm{n}=6)$, with a range of $12.54-14.62 \mathrm{~mm}$. Thus, on average, the SG was only $41 \%$ as long as the OC, and this ratio was fairly similar in cochleae of different sizes. Direct morphometric analysis demonstrated that Rosenthal's canal terminated and no SG cell somata were found directly radial to the most basal $2 \%$ and the most apical $11 \%$ of the OC, on average. 
Fig. 2. Frequency-matched points along the OC and SG were determined by tracing the trajectories of radial nerve fibers. These data are plotted here for the $6 \mathrm{co}-$ chleae in our data set. A good correlation between percentage length along the $\mathrm{OC}$ and percentage length along the SG was demonstrated $\left(r^{2}>0.99\right)$. The data were well fit by the cubic function shown in the graph. Mean location for $\mathrm{V}$ is indicated by arrow (see text).

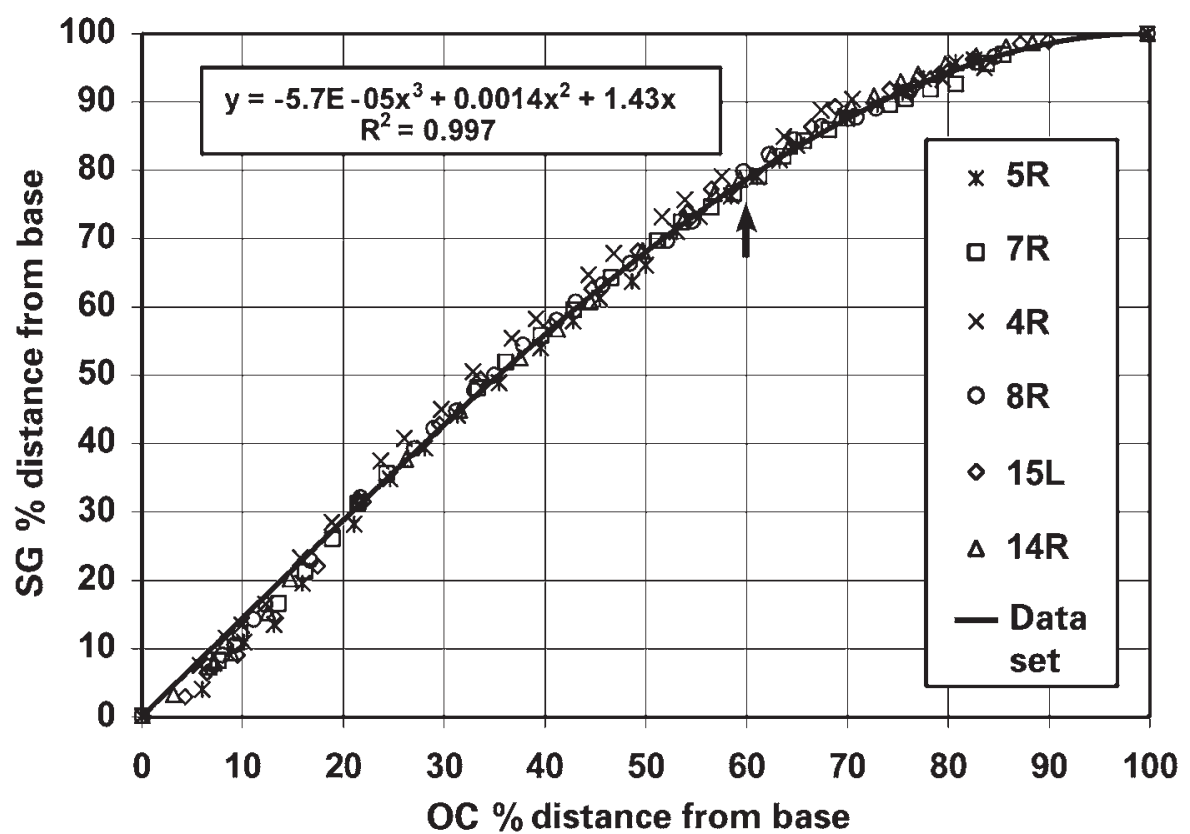

Table 1. Summary of measurements in 7 cochleae

\begin{tabular}{|c|c|c|c|c|c|c|c|c|}
\hline & \multicolumn{8}{|c|}{ Specimen } \\
\hline & $7 \mathrm{R}$ & $4 \mathrm{R}$ & $5 \mathrm{R}$ & $8 \mathrm{R}$ & $15 \mathrm{~L}$ & $14 \mathrm{R}$ & $6 \mathrm{R}$ & mean \\
\hline OC length, mm & 30.5 & 32.31 & 33.44 & 36.16 & 32.45 & 36.87 & 31.41 & 33.31 \\
\hline SG length, mm & 12.54 & 14 & 14.2 & 14.58 & 13.44 & 14.62 & NA & 13.90 \\
\hline SG length/OC length & 0.41 & 0.43 & 0.42 & 0.4 & 0.41 & 0.4 & NA & 0.41 \\
\hline Percent OC basal without SG & 1.31 & 1.98 & 3.47 & 1.38 & 2.13 & 1.84 & NA & 2.02 \\
\hline Percent OC apical without SG & 11.64 & 9.22 & 11.33 & 10.26 & 11.37 & 12.99 & NA & 11.14 \\
\hline Percent OC length at V & 59.08 & 59.36 & 64.74 & 55.56 & 61.14 & NA & 60.01 & 59.98 \\
\hline Frequency at $\mathrm{V}, \mathrm{Hz}$ & 1051 & 1035 & 764 & 1273 & 937 & NA & 998 & 1000 \\
\hline
\end{tabular}

Data collected in 7 cochleae indicate relatively large intersubject variability in OC and SG lengths, but a fairly consistent ratio between the lengths of the two structures. Dissection error resulted in damage to the basal SG in specimen 6R and precluded measuring SG length. The marker in the stria vascularis for $\mathrm{V}$ was lost during processing of the cochlea and could not be identified in specimen $14 \mathrm{R}$.

Frequency-matched coordinates on the $\mathrm{OC}$ and SG were determined by directly tracing the trajectories of radial nerve fibers at numerous points along the cochlear spiral in the digital images. These data are presented in figure 2. Percentage length along the SG was found to relate consistently and predictably to percentage length along the OC, with minimal intrasubject and intersubject variability. The data were fit by a cubic function that was constrained to begin at the point $\mathrm{x}=0, \mathrm{y}=0$, to end at $\mathrm{x}=100, \mathrm{y}=100$ and to be monotonic: $\mathrm{y}=-5.7 \mathrm{E}-05 \mathrm{x}^{3}+$
$0.0014 x^{2}+1.43 x$, where $y=$ percentage distance from base of the SG and $\mathrm{x}=$ percentage distance from base of the OC. Since Greenwood's function defines frequency for the OC, SG frequency can then be calculated.

As anticipated, the frequency-position function for the SG is quite different from that of the basilar membrane for a large proportion of the cochlea, and particularly for the apical third of the cochlea. During cochlear dissections, an anatomical reference point was created by marking the site on the stria vascularis of the upper bas- 
al coil nearest to the center of the vestibule. This marker, which was termed $\mathrm{V}$, was maintained throughout subsequent processing and embedding of each cochlea. The mean location of $\mathrm{V}$ in our study group was about $60 \%$ from the basal end of the OC (table 1), but on average this point was located $78 \%$ from the base of the SG, as indicated by the arrow in figure 2 . It is interesting to note that the mean location for $\mathrm{V}$ is very close to the site where the radial nerve fibers begin to lose their radial orientation and begin to take a progressively more tangential course into the SG (mean, $60.8 \% \pm 3.85 \mathrm{SD}$ ).

The represented frequency at our anatomical reference point $\mathrm{V}$, as calculated using the Greenwood function, had a mean value of $1000 \mathrm{~Hz}$, but it varied across quite a wide range from 1275 to $760 \mathrm{~Hz}$ for the specimens in our study. This landmark (point on basal coil nearest to the center of the vestibule) can be imaged in living subjects, so these data give us a preliminary estimate of the individual variability in the cochlear place frequency for a CI electrode positioned at V.

The relationship shown in figure 2 between distance along the $\mathrm{OC}$ and distance along the SG allows us to use Greenwood's equation to provide the first estimates of critical band distance in the human SG. Unlike critical band distance for the OC, which remains constant from base to apex, the critical band distance estimated along the SG varies as a function of position, and becomes progressively narrower from base to apex. Specifically, a critical band distance for the OC is suggested by Greenwood to equal $1 / 35$ the basilar membrane length and is constant throughout the cochlea [Greenwood, 1990]. For our average cochlea, the OC was $33.31 \mathrm{~mm}$ long and the critical band distance would be $0.95 \mathrm{~mm}$ (1/35th of 33.31). SG critical band distances were estimated throughout the cochlea by using the function derived in figure 2 to estimate the length of ganglion associated with each millimeter of OC, as calculated for our mean SG length of $13.9 \mathrm{~mm}$. Specifically, near the cochlear base one critical band distance on the $\mathrm{OC}(0.95 \mathrm{~mm})$ corresponds to a distance of about $0.6 \mathrm{~mm}$ in the SG, whereas in the lower middle turn $(24 \mathrm{~mm}$ from the base, $\approx 500 \mathrm{~Hz}$ ) a critical band distance on the OC is still the same $(0.95 \mathrm{~mm})$ but the critical band distance in the SG is reduced to approximately $0.32 \mathrm{~mm}$. This compression of critical band distance within the SG is particularly pronounced in the apical $40 \%$ of the cochlea (frequencies of $1 \mathrm{kHz}$ and lower), where the radial nerve fibers begin to take an increasingly tangential course from the OC into the SG.

\section{Discussion and Conclusions}

Great intersubject variability was observed in both basilar membrane and SG lengths, especially in consideration of the limited number of cochlear specimens included in this series. OC length ranged from 30.5 to $36.87 \mathrm{~mm}$ (mean, $33.31 \mathrm{~mm}$ ). This finding is consistent with numerous prior reports indicating great variability in human cochlear length [Kawano et al., 1996; Ketten et al., 1998; Skinner et al., 2002; Bredberg, 1968; Ulehlova et al., 1987; Hardy, 1988]. The SG was $40-43 \%$ as long and ranged in length from 12.54 to $14.62 \mathrm{~mm}$ (mean, $13.9 \mathrm{~mm})$.

The represented frequency at our selected anatomical landmark $\mathrm{V}$ (the point on the basal turn closest to the center of the vestibule), as calculated using the Greenwood function, had a mean value of $1000 \mathrm{~Hz}$, although it varied across quite a wide range from 1275 to $760 \mathrm{~Hz}$ for the specimens in our study. Our data suggest that in order to reach this $1000-\mathrm{Hz}$ position in the average cochlea, a $\mathrm{CI}$ electrode carrier which is positioned under the OC would have to place a stimulating contact at a point $20 \mathrm{~mm}$ from the extreme base of the OC (i.e. $60 \%$ of $33.31 \mathrm{~mm}$ ). This is probably a maximum estimate of the required insertion length to reach this point, because most surgeons insert the CI electrodes through a cochleostomy anterior to the round window and therefore several $\mathrm{mm}$ from the basal end of the OC.

On the other hand, some current CIs are designed to position stimulation sites facing the modiolus and as close as possible to the SG. The implicit assumption is that the SG is the target for electrical stimulation. In this case, if a CI electrode carrier achieved an ideal perimodiolar position directly adjacent to the SG, the approximate insertion depth required to reach $\mathrm{V}$ would be $78 \%$ of $13.9 \mathrm{~mm}$ or only $10.84 \mathrm{~mm}$ in the average cochlea, or $11.4 \mathrm{~mm}$ for the longest SG in our data set. Moreover, the maximum length of the SG in our series was less than $15 \mathrm{~mm}$. These data suggest that an ideally positioned perimodiolar CI electrode targeting the SG probably would have to be inserted to a maximum depth of only about $15 \mathrm{~mm}$ in order to position stimulating contacts adjacent to the entire length of Rosenthal's canal. However, it should be noted that our measurements of SG length were made at the center of Rosenthal's canal. The exact distance along the modiolar wall to the apical terminus of the SG and the extent of intersubject variability must be determined empirically in future studies. This is an important issue, since shorter insertion depths are less likely to cause trauma to the cochlea [Wardrop et al., 
2005a, b], thus optimizing clinical outcome. Particularly in the case of electroacoustic stimulation, shorter insertion depths may improve the chances for preservation of residual hearing.

Normalized length along the SG (\% distance from the base) was highly correlated to normalized length along the OC, and was best fit by a cubic function. Since the Greenwood function allows calculation of frequency for the $\mathrm{OC}$, this new equation provides a frequency-position function for the human SG. However, because of the individual variability in cochlear size, accurate estimates of frequency for $\mathrm{CI}$ electrodes positioned at different points along the OC or SG in living subjects will require a method of estimating OC or SG length in the imaging studies. We hypothesize that measurements of the basal turn diameter [e.g., see Escudé et al., this issue, pp. 27-33] may provide a reliable indication of overall cochlear size and may correlate to OC or SG length. Additional temporal bone studies are required to test this hypothesis.

The SG frequency-position function allowed us to calculate critical band distances for the SG. Unlike critical bandwidths for the OC, which remain constant throughout the cochlea, estimated critical band distances in the SG diminish systematically from base to apex. One intriguing implication of these calculations is that when individual electrodes are spaced at uniform intervals along the implant carrier, which is the case in most contemporary CI designs, the frequency shift between adjacent electrodes is greatest at the base and much more compressed for apical electrodes. An exception to the usual uniform spacing of electrodes is seen in the Contour Advance electrode which has reduced spacing at the apical end, but additional research is required to determine how this spacing would 'fit' the SG critical band distance compression with different insertion depths and in different individuals. Finally, it should be noted that electrode spacing is an extremely complicated issue with many other factors such as spread of excitation and channel interaction having an impact on optimum electrode spacing and stimulation patterns.

The longitudinal positions of CI electrodes in the cochlea have been correlated with pitch perception as well as with threshold, growth of loudness and speech intelligibility [Skinner et al., 2002]]. As subjects with greater residual hearing and better SG survival receive implants and advances in CI design permit more spatially precise stimulation, an accurate frequency map for the SG may provide a basis for better matching subjects' speech processor maps to the appropriate frequencies of CI stimulation sites, potentially increasing clinical benefits. In electroacoustic stimulation, more accurate estimates of the frequency-place function in individual subjects may allow investigators to determine preoperatively the precise length of an implant required to provide electrical hearing over the range of frequencies optimum for that individual, based upon the frequency range of their residual hearing.

\section{Acknowledgements}

We would like to thank Ben Bonham, $\mathrm{PhD}$, for his help in optimizing the frequency-position function for the spiral ganglion. This research was supported by the U.S. National Institutes of Health, National Institute on Deafness and Other Communications Disorders, contract N01-DC-3-1006 and by a Doris Duke Clinical Research Fellowship to D.S. Dr. Leake's expenses to attend the 1st International Electro-Acoustic Workshop, Toulouse, December 2005, were reimbursed by Cochlear Corporation.

\section{References}

Bredberg G: Cellular pattern and nerve supply of the human organ of Corti. Acta Otolaryngol 1968;236(suppl):1-135.

-Escudé B, James C, Deguine O, Cochard N, Eter E, Fraysse B: The size of the cochlea and predictions of insertion depth angles for cochlear implant electrodes. Audiol Neurotol 2006; 11(suppl 1):27-33.

-Greenwood DD: A cochlear frequency-position function for several species -29 years later. JASA 1990;87:2592-2605.

- Hardy M: The length of the organ of Corti in man. Am J Anat 1988;62:291-311.

-Kawano A, Seldon HL, Clark GM: Computeraided three-dimensional reconstruction in human cochlear maps: measurement of the lengths of organ of Corti, outer wall, inner wall, and Posenthal's canal. Ann Otol Rhinol Laryngol 1996;105:701-709.

Ketten D, Skinner MW, Wand G, Vannier MW Gates GA, Neely JG: In vivo measures of cochlear length and insertion depth of nucleus cochlear implant electrode arrays. Ann Otol Rhinol Laryngol 1998;107:1-16.

Skinner MW, Ketten DR, Holden LK, Harding GW, Smith PG, Gates GA, Neely JG, Kletzker GR, Brunsden B, Blocker B: CT-derived estimation of cochlear morphology and electrode array position in relation to word recognition in Nucleus-22 recipients. JARO $2002 \cdot 3 \cdot 332-350$
Ulehlova L, Voldrich L, Janisch R: Correlative study of sensory cell density and cochlear length in humans. Hear Res 1987;28:149-151.

-Wardrop P, Whinney D, Rebscher SJ, Luxford W, Leake PA: A temporal bone study of insertion trauma and intracochlear position of cochlear implant electrodes. II. Comparison of Spiral Clarion ${ }^{\mathrm{TM}}$ and HiFocus II ${ }^{\mathrm{TM}}$ electrodes. Hear Res 2005b;203:68-79.

-Wardrop P, Whinney D, Rebscher SJ, Roland JT Jr, Luxford W, Leake PA: A temporal bone study of insertion trauma and intracochlear position of cochlear implant electrodes. I. Comparison of Nucleus banded and Nucleus Contour $^{\mathrm{TM}}$ electrodes. Hear Res 2005a;203: 54-67. 